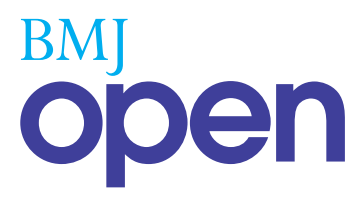

\title{
Ulcer healing time and antibiotic treatment before and after the introduction of the Registry of Ulcer Treatment: an improvement project in a national quality registry in Sweden
}

\author{
Rut F Öien, Henrik W Forssell
}

To cite: Öien RF, Forssell HW. Ulcer healing time and antibiotic treatment before and after the introduction of the Registry of Ulcer Treatment: an improvement project in a national quality registry in Sweden. BMJ Open 2013;3:e003091. doi:10.1136/bmjopen-2013003091

- Prepublication history for this paper is available online. To view these files please visit the journal online (http://dx.doi.org/10.1136/ bmjopen-2013-003091).

Received 22 April 2013 Revised 20 July 2013 Accepted 24 July 2013

Blekinge Wound Healing Centre, Blekinge Centre of Competence, Karlskrona, Sweden

Correspondence to Dr Rut F Öien; rut.oien@|tblekinge.se

\section{ABSTRACT}

Objectives: To investigate changes in ulcer healing time and antibiotic treatment in Sweden following the introduction of the Registry of Ulcer Treatment (RUT), a national quality registry, in 2009.

Design: A statistical analysis of RUT data concerning the healing time and antibiotic treatment for patients with hard-to-heal ulcers in Sweden between 2009 and 2012.

Setting: RUT is a national web-based quality registry used to capture areas of improvement in ulcer care and to structure wound management by registering patients with hard-to-heal leg, foot and pressure ulcers. Registration includes variables such as gender, age, diagnosis, healing time, antibiotic treatment, and ulcer duration and size.

Population: Every patient with a hard-to-heal ulcer registered with RUT between 2009 and $2012(n=1417)$ was included.

Main outcome measures: Statistical analyses were performed using Stata V.12.1. Healing time was assessed with the Kaplan-Meier analysis and adjustment was made for ulcer size. A log-rank test was used for equality of survivor functions.

Results: According to the adjusted registry in December 2012, patients' median age was 80 years (mean 77.5 years, range $11-103$ years). The median healing time for all ulcers, adjusted for ulcer size, was 146 days ( 21 weeks) in 2009 and 63 days (9 weeks) in $2012(p=0.001)$. Considering all years between 2009 and 2012, antibiotic treatment for patients with hard-toheal ulcers was reduced from $71 \%$ before registration to $29 \%$ after registration of ulcer healing $(p=0.001)$.

Conclusions: Healing time and antibiotic treatment decreased significantly during 3 years after launch of RUT.

\section{INTRODUCTION}

Wound management not only consumes time and money, but also ulcers and their treatment reduce the quality of life for the affected patients. ${ }^{1-3}$

\section{ARTICLE SUMMARY}

Article focus

- The aim of this study was to analyse the data from the Swedish Registry of Ulcer Treatment (RUT) to detect any differences in healing time and antibiotic treatment between 2009 and 2012.

Key messages

- Median healing time for all ulcers (adjusted for ulcer size) decreased significantly from 146 days in 2009 to 63 days in 2012.

- Median healing time for venous ulcers (adjusted for ulcer size) decreased significantly from 120 days in 2009 to 69 days in 2012.

- Antibiotic treatment for patients with hard-to-heal ulcers was reduced from $71 \%$ before registration to $29 \%$ while using RUT.

Strengths and limitations of this study

- The data covers every patient registered with RUT during 2009-2012.

- RUT covers wound management in primary care, community care, private care and in-patient hospital care throughout Sweden.

- RUT provides a reliable diagnosis, adequate strategies for ulcer care and a structured follow-up of ulcer healing.

- One limitation is that RUT is still in the process of being implemented, which means that in some areas of Sweden every patient with a hard-to-heal ulcer is registered, while registration of patients in other areas is only partial.

Patients with hard-to-heal ulcers have long been considered a neglected patient population. Many are treated without diagnosis, and they consequently received suboptimal ulcer care. Overuse of oral antibiotics in these patients $(68-78 \%)^{1}{ }^{4-6}$ is mostly due to the absence of diagnosis or inadequate clinical assessment of ulcer infections. ${ }^{67}$ The lack of continuity in ulcer care and also the lack of 
team-work between health professionals in this field has further contributed to the overuse of antibiotics. ${ }^{8}$ In Sweden, the absence of national guidelines for medical ulcer care has had a negative impact on wound management.

Structured wound management based on accurate diagnosis leads to effective treatment and consequently to decreased prevalence, care time and costs. ${ }^{9}$ For this reason, the Swedish RUT ${ }^{10}$ was started in May 2009. Its purpose is to assess physician diagnoses of ulcers, furnish a structured checklist for the medical staff for optimal treatment and identified areas of improvement in wound management.

The focus of this study was to investigate whether ulcer healing time and antibiotic treatment have been affected by the registry. We aimed to analyse data from RUT since the time the registry was been introduced nationally (2009-2012).

\section{National registries}

In recent decades, a system of national quality registries has been established in the Swedish health and medical services, ${ }^{11}$ covering different areas of medicine. There are currently 73 registries that received central funding (http://www.skl.se).

The ambition of the Swedish national quality registries is to gather data on diagnoses, symptoms, interventions and treatment outcomes in order to give a continuous systematic evaluation of medical practice.

The majority of the Swedish national quality registries have been developed by physicians with special interest in a research field, in order to bring about quality improvement to healthcare for a specific medical problem.

\section{The Registry of Ulcer Treatment}

For the past 25 years, healthcare professionals in Blekinge have been focused on quality improvement and clinical research within the field of wound management. ${ }^{19}$ This tradition of research linked to clinical practice led to the establishment of the Blekinge Wound Healing Centre in 2003. This is a general practitioner (GP)-led, primary carebased specialist centre covering the treatment and follow-up of the majority of ulcer patients across the county (150000 inhabitants). It offers a structured team management of ulcer care with an emphasis on diagnosis, documentation and treatment. The GP in charge of the centre is the first author of this study (RFÖ).

The experience of our daily practice combined with research results, soon made it obvious that there was a need for a structured programme for wound management to guarantee optimal treatment. RUT was started in Blekinge County by RFÖ, the registry manager, who then developed the registry further and launched it nationwide.

RUT was the first national registry in primary care. It is web-based and the participating units use the registry as a checklist for ulcer assessment and a base for quality improvement in their units.
The data must be collected in a uniform manner to capture the scale of wound care, as has been previously pointed out. ${ }^{12}$ The conventional approach is to record details of ulcer care such as treatment strategies, dressings, antibiotics, analgesics, investigations, hospital admissions and surgical interventions at each patient contact over the period from first presentation to wound healing. ${ }^{12}$ These details are noted in the patient's medical record to support decisions regarding the care for that individual patient.

Registration in RUT usually takes place at the first patient contact, and follow-up can be carried out when all the mandatory variables are registered. Each unit has access only to data of its own patients; these can be retrieved online at any time, and used to compare the unit's quality of wound management with that across the country. By using accumulated data for the whole country, areas of improvement can be highlighted.

To capture the situation of patients with pressure ulcers, during the study period, the pressure ulcer section of the registry was further developed. To improve our coverage of community units (where these patients are mostly treated), we have established cooperation with another Swedish national quality registry on pressure ulcer prevention.

This could in the future give a more accurate picture of the pressure ulcer prevention linked to either pressure ulcer healing or negative clinical events, such as death.

Registering with RUT has been mandatory in Blekinge County since 2012 and today some of the larger national dermatological departments are likewise obliged to register with RUT.

\section{METHODOLOGY}

\section{Study population and variables}

During the study period we had frequent meetings with the participating units, at which nurses and physicians provided feedback on RUT. Having had access to the structure of the registry, they commented on how the registry had made a difference in their approach to wound management. Their new focus was on adequate diagnosis, continuity of care by nominating a responsible ulcer nurse for every patient and follow-up to ulcer healing. They saw the documented shorter time to healing as an acknowledgment of their efforts to introduce improved wound management.

Another area of improvement was the reduction in antibiotic treatment. Since the variable for antibiotic treatment before and after registration is mandatory, the participants' attention was drawn to antibiotic use. They acknowledged the reduction in oral antibiotic treatment for their patients as a success for their unit and an improvement of the patients' quality of life.

During the study period, 160 patients were registered in 2009, 348 patients in 2010, 400 in 2011 and 509 in 2012, making a total of 1417 patients nationwide by 2012 . 
The RUT registers patients with hard-to-heal leg, foot or pressure ulcers on two occasions. The first registration, assessment of the ulcer diagnosis, is to guarantee optimal treatment. The second registration is at follow-up; that is, at the point where the ulcer has healed or a negative clinical event such as amputation or death has occurred. Every patient with a non-healed ulcer remains in the registry until follow-up is completed.

At the first registration the following variables are recorded: social security number, gender, age, date of diagnosis, profession or former profession, smoking habits, civil status, number of children, mobility, exercise habits and body mass index. The patient's social security number is linked and matched to the population statistics at the Council for Official Statistics of Sweden.

To guarantee continuity of care there is a mandatory variable containing the name of the nurse responsible for ulcer care, facilitating follow-up of every patient to complete ulcer healing. This nurse is often the person responsible for registering the patient with RUT.

Other details taken include whether the ulcer is new or recurrent, current or earlier concomitant diseases, current medication, particularly analgesics and antibiotics; and ulcer-related pain. The patient history focuses on the following variables: deep vein thrombosis, varicose veins, arterial or venous surgery, history of recurrent leg ulcers and ulcer localisation (foot, leg or sacrum/hip; lateral or medial). The ulcer size is measured by a digital planimeter (Visitrak, manufactured in the UK for Smith \& Nephew Medical Limited, Hull HU3 2BN) and the number of ulcers is noted.

The patient's arterial circulation is assessed by palpating the arteria dorsalis pedis and arteria tibialis posterior and measuring the ankle-brachial pressure index with a hand-held Doppler (manufactured by Histolab, Gothenburg, Sweden). The Doppler is also used for measuring deep or superficial venous insufficiency (vena saphena magna, vena saphena parva and vena poplitea).

The diagnosis is determined from these variables in combination with the clinical examination. The following ulcer diagnoses are used: venous ulcer, arterial ulcer, venous-arterial ulcer, diabetic foot ulcer, pressure ulcer, traumatic ulcer, ulcer due to inflammatory vessel diseases such as vasculitis, and other diagnoses (eg, pyoderma gangrenosum). The strategy for wound management includes dressings, care for the skin surrounding the ulcer and treatment for oedema. A photo gallery is linked to the registry for visualisation of the healing process.

The second registration (at follow-up) includes date of healing, healing time, estimated number of weekly dressing changes throughout healing, compression therapy, treatment with antibiotics, pain relief, the most frequently used dressing material and whether advice was given on smoking cessation, exercise and diet. Adverse events are also recorded, such as amputation, venous or arterial surgery, pinch grafting and death.

\section{Data analysis}

The statistical analysis was performed using Stata V.12.1 (StataCorp LP, College Station, Texas, USA). Continuous variables were expressed as mean values $( \pm \mathrm{SD})$ and compared using two-sample Student's t tests. Group comparisons for categorical variables were performed using Pearson's $\chi^{2}$ tests. Healing time was assessed using the Kaplan-Meier analysis and adjustment was made for ulcer size. A log-rank test was used for equality of survivor functions. The $p$ value $<0.05$ was considered statistically significant.

\section{RESULTS}

\section{Healing time}

Basic data from the adjusted registry in December 2012 showed a population $(\mathrm{n}=1417)$ with a median age of 80 years (mean age 77.5 years), ranging from 11 to 103 years.

The majority of the patients were women $(60 \%)$. The median ulcer duration was 12 weeks (mean 117 weeks), ranging from 1 week to 46 years, and the median ulcer size at inclusion in RUT was $3 \mathrm{~cm}^{2}$ (mean $12 \mathrm{~cm}^{2}$, range $0.05-600 \mathrm{~cm}^{2}$ ).

The participating units covered primary care $(50 \%)$, community care $(4 \%)$, hospital care $(22 \%)$, wound healing centres (22\%) and private caregivers (2\%). Patients from Blekinge constituted $39 \%$ of all patients.

Figure 1 illustrates the median healing time, adjusted for ulcer size, from 2009 to 2012. The median healing time was 146 days (21 weeks) for all ulcers in 2009 and 63 days ( 9 weeks) for all ulcers in $2012(\mathrm{p}=0.001)$.

Negative pressure wound therapy was introduced in Europe in 1997, and has been used in Swedish primary healthcare since 2006. During the study period, this technique was used only in $1.3 \%$ of cases.

Figure 2 gives the median healing time for venous ulcers, adjusted for ulcer size, from 2009 to 2012. The median healing time for these ulcers was 120 days (17 weeks) in 2009 and 69 days (10 weeks) in 2012

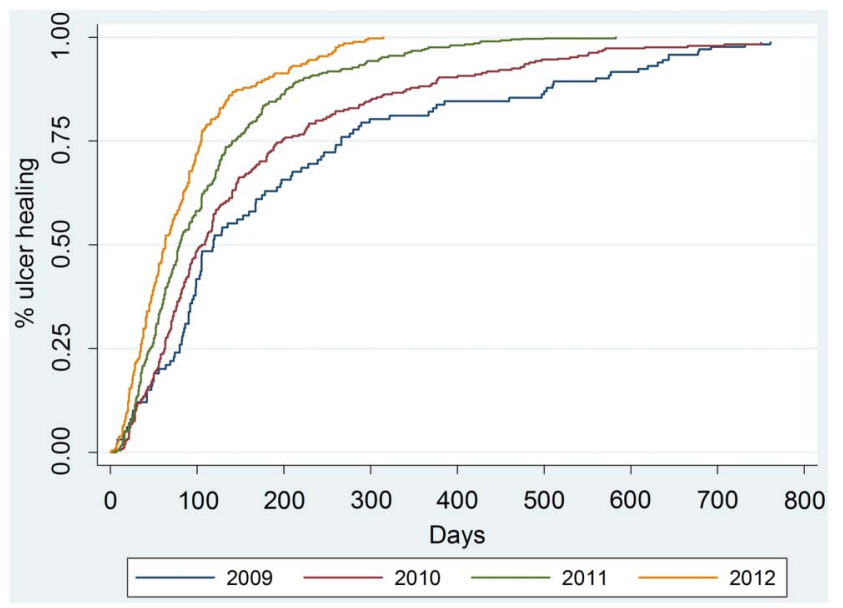

Figure 1 Ulcer healing time, 2009-2012. Figures adjusted for ulcer size. 


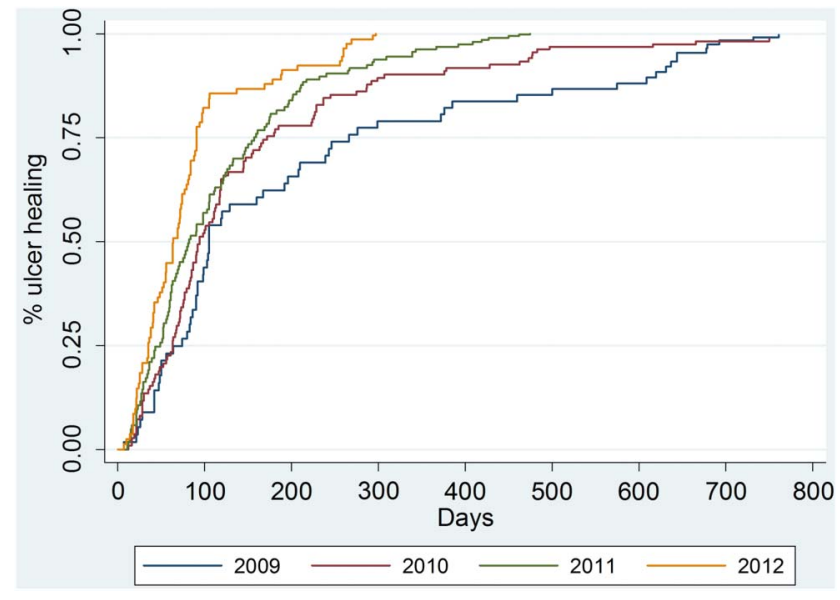

Figure 2 Venous ulcer healing time, 2009-2012. Figures adjusted for ulcer size.

$(\mathrm{p}=0.001)$. Compression therapy was used in $87.3 \%$ of venous ulcers in 2009 and $88.9 \%$ in 2012. In some areas of Sweden, every venous ulcer $(100 \%)$ was treated with compression therapy both in 2009 and in 2012.

\section{Antibiotic treatment}

Figure 3 illustrates antibiotic treatment from 2009 to 2012. In $2009,76 \%$ of patients were treated with antibiotics before registration, compared with $24 \%$ after registration. In 2012, the corresponding figures were $73 \%$ before and $27 \%$ after registration. These differences were significant in both years $(\mathrm{p}=0.001)$.

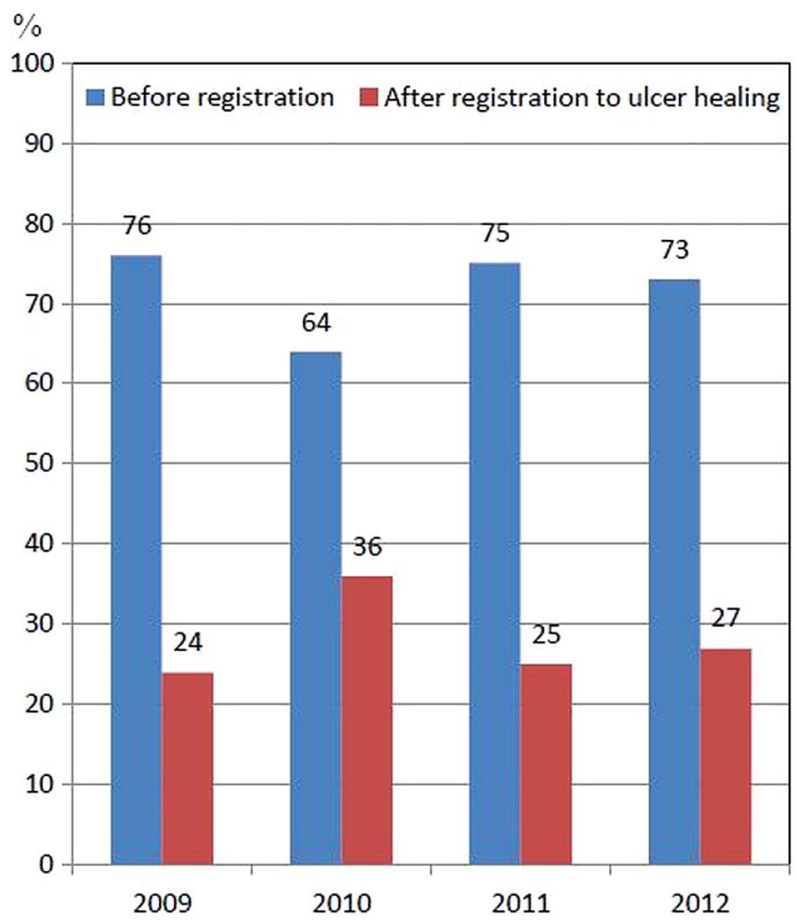

Figure 3 Antibiotic treatment before registration in Registry of Ulcer Treatment (RUT) for the years 2009-2012, compared with antibiotic treatment between registration and ulcer healing for the same years.
Considering all years between 2009 and 2012, the antibiotic treatment for patients with hard-to-heal ulcers in the registry nationwide was reduced from $71 \%$ before registration to $29 \%$ after registration $(p=0.001)$.

\section{DISCUSSION}

The principal finding in this study was the significantly reduced healing time for hard-to-heal ulcers registered with RUT, from 146 days (21 weeks) in 2009 to 63 days (9 weeks) in 2012. This reduction in healing time seems to be due to structured wound management, based on accurate diagnosis, continuity of care by nominating a responsible nurse for every patient with ulcer and follow-up to healing, all of which factors were facilitated by RUT.

Although topical antimicrobial therapy for local ulcer infection, such as iodine, silver, honey and polyhexamethylene biguanide (PHMB), has become more firmly established, no further innovative dressings or devices were introduced for wound management on the Swedish market during the study period. Negative pressure wound therapy was used in less than $1.5 \%$ of all cases during the study, but is now more widespread in clinical practice. There was some development of services, such as the introduction of smaller wound healing centres based on the model of the Blekinge Wound Healing Centre.

Healing time is the one important endpoint in wound management. ${ }^{13}$ Earlier researchers found a median healing time of 20-43 weeks while following patients over a period of 12 months. ${ }^{13}$ Some researchers have noted a healing rate of $83 \%$ in 30 weeks, ${ }^{14}$ while others have reported that $62 / 90(69 \%)$ of venous leg ulcers healed within 12 weeks. ${ }^{15}$ Moffatt et al ${ }^{16}$ found that $70 \%$ of venous ulcers healed after 48 weeks of treatment; they also noted that much of the evidence on healing rates is drawn from the results of randomised controlled trials. These trials typically achieve 24-week healing rates in excess of $60 \%$, but may not reflect the complex issues faced in clinical practice. ${ }^{16}$

Another important finding in the present study was the low proportion of patients (24-27\%) given antibiotics between registration and ulcer healing; that is, in patients with a diagnosis and adequate treatment. This can be compared with earlier findings of $68-78 \%^{15}$ for patients in primary care. Between 2009 and 2012, antibiotic treatment for patients with hard-to-heal ulcers in the registry nationwide was reduced from $71 \%$ before registration to $29 \%$ between registration and ulcer healing $(\mathrm{p}=0.001)$.

There is still a high rate of oral antibiotic treatment for patients outside the registry, which may be explained by the fact that these patients do not receive continuity of care or treatment by a specialised team, where topical antimicrobial treatment is the golden standard for treating local ulcer infection.

The use of topical antimicrobial dressings could be one explanation for the reduction in antibiotic treatment. This issue is being addressed in an ongoing research study within the frame of RUT. 
A further explanation for the recent low rate of antibiotic treatment and reduced healing time could be that RUT focuses on stable doctor-patient relationships. Previous research has shown the advantages of a structured organisation for leg ulcer care. ${ }^{17} 18$ Petursson $^{8}$ argues that lack of continuity in medical care is the main reason why GPs prescribe antibiotics in a "nonpharmacological' manner.

The ulcer patient's right to receive optimal treatment has been limited in recent decades, owing to the lack of diagnosis and low continuity in ulcer care. ${ }^{1} \quad 16 \quad 1920$ Changes in dressing over weeks, months and even years have been carried out, often without a proper diagnosis. ${ }^{1}$ It is well known that understanding the aetiology of leg ulceration is a pre-requisite for a systematic clinical assessment as a base for appropriate wound management. ${ }^{121415}$ RUT meets these requirements.

A large proportion of the patient population $(39 \%)$ in the registry was concentrated in Blekinge County. This could be considered a bias, as the registry was developed in Blekinge County before being expanded to the whole country. Hence the majority of patients were treated in primary care reflecting the true situation in Sweden.

The healthcare system requires information on the burden of care in order to inform decisions on the needs of the population and the allocation of resources. ${ }^{16}$ RUT comprises a structured and practical methodology which can be used at any level in the healthcare system.

Nationwide implementation of RUT has not yet been fully achieved. One area for future research is to investigate differences in the results of ulcer care between areas in Sweden where RUT is used and areas where it is not used.

Another further research issue concerns the mapping of all pressure ulcers, such as ulcers in younger patients with neurological diseases and in palliative care patients. RUT is an appropriate basis for such a study, as it has a special section for pressure ulcers.

Since March 2013, the registry has included 1438 patients. The role of the registry manager and the steering group is to show the staff how data from the registry can be used to improve ulcer care. We focus on documentation, treatment, education, research and economic analyses to guarantee improvement of health outcomes nationwide. Our ultimate aim is twofold: to serve as a basis for national guidelines and for the registry to be implemented internationally.

\section{CONCLUSION}

The findings from this study illustrate the immediate impact of RUT as an improvement project within wound management, resulting in significantly reduced healing time from 146 days (21 weeks) in 2009 to 63 days (9 weeks) in 2012. Antibiotic treatment was reduced from $71 \%$ before registration to $29 \%$ between registration and ulcer healing. The results also demonstrate the potential for improved wound management while using a national quality registry for structured ulcer care.
Acknowledgements This study was performed in the interests of the steering group for the Swedish Registry of Ulcer Treatment (RUT).

Contributors RFÖ led the research project and played the main role in the research design. HWF contributed to the data analysis and assisted in the research design and interpretation of results.

Funding This study was partly funded by the Council of Sciences in Blekinge County.

\section{Competing interests None.}

Ethics approval The Lund Ethical Review Board considered the study to be a valuable quality improvement study, so no further ethical approval was required.

Provenance and peer review Not commissioned; externally peer reviewed.

Data sharing statement No additional data available.

Open Access This is an Open Access article distributed in accordance with the Creative Commons Attribution Non Commercial (CC BY-NC 3.0) license, which permits others to distribute, remix, adapt, build upon this work noncommercially, and license their derivative works on different terms, provided the original work is properly cited and the use is non-commercial. See: http:// creativecommons.org/licenses/by-nc/3.0/

\section{REFERENCES}

1. Öien RF, Håkansson A, Ovhed I, et al. Wound management for 287 patients with chronic leg ulcers demands 12 full-time nurses. Leg ulcer epidemiology and care in a well-defined population in Southern Sweden. Scand J Prim Health Care 2000;18:220-5.

2. Lindholm C, Bjellerup M, Christensen OB, et al. Quality of life in chronic leg ulcer patients: an assessment according to the Nottingham Health Profile. Acta Derm Venereol 1993;73:440-3.

3. Anon. Evidence-based prescribing of advanced wound dressings for chronic wounds in primary care. MeRec Bull 2010;1:1-7.

4. Wiström J, Lindholm C, Melhus A, et al. Infections and treatment in chronic leg ulcers: the use of antibiotics is too excessive, restrictive prescription is recommended. Lakartidningen 1999;6:42-6 [in Swedish]

5. André M, Eriksson M, Odenholt I. Treatment of patients with skin and soft tissue infections: results from the STRAMA survey of diagnoses and prescriptions among general practitioners. Lakartidningen 2006;103:3165-7 [in Swedish].

6. Öien RF, Åkesson N. Bacterial cultures, rapid strep test and antibiotic treatment in infected hard-to-heal ulcers in primary care. Scand J Prim Health Care 2012;30:254-8.

7. European Wound Management Association (EWMA) Position document: management of wound infection. London: MEP, 2006.

8. Petursson P. GPs' reasons for "non-pharmacological" prescribing of antibiotics: a phenomenological study. Scand J Prim Health Care 2005;23:120-5.

9. Öien RF, Ragnarson Tennvall G. Accurate diagnosis and effective treatment of leg ulcer reduce prevalence, care time and costs. $J$ Wound Care 2006;15:259-62.

10. Öien RF. RUT (Register of Ulcer Treatment) - a winning concept for both patients and the health care sector. EWMA J 2009;9:41-4.

11. Lundström M, Albrecht S, Serring I, et al. Handbook for establishing quality registries. Karlskrona, Sweden: EyeNet Sweden, 2005.

12. Harding K, Posnett J, Vowden K. A new methodology for costing wound care. Int Wound J 2012. doi: 10.1111/iwj.12006

13. Morrell CJ, Walters SJ, Dixon S, et al. Cost effectiveness of community leg ulcer clinics: randomised controlled trial. BMJ 1998;316:1487-91.

14. Rybak Z, Franks PJ, Krasowski G, et al. Strategy for the treatment of chronic leg wounds: a new model in Poland. Int Angiol 2012;31:550-6.

15. Hjerppe A, Saarinen JP, Venermo MA, et al. Prolonged healing of venous leg ulcers: the role of venous reflux, ulcer characteristics and mobility. J Wound Care 2010;19:474, 476, 478 passim.

16. Moffatt CJ, Doherty DC, Smithdale R, et al. Clinical predictors of leg ulcer healing. Br J Dermatol 2010;162:51-8.

17. Moffatt CJ, Franks PJ, Oldroyd $M$, et al. Community clinics for leg ulcers and impact on healing. BMJ 1992;305:1389-92.

18. Kjaer ML, Sorensen LT, Karlsmark T, et al. Evaluation of the quality of venous leg ulcer care given in a multidisciplinary specialist centre. $J$ Wound Care 2005;14:145-50.

19. Törnvall E, Wilhelmsson S. Quality of nursing care from the perspective of patients with leg ulcers. J Wound Care. 2010;19:388-95.

20. Edwards H, Finlayson K, Courtney M, et al. Health service pathways for patients with chronic leg ulcers: identifying effective pathways for facilitation of evidence based wound care. BMC Health Serv Res 2013;13:86 